\title{
Um método de pontos interiores para resolução de problemas lineares discretos mal-postos
}

\author{
Emídio Santos Portilho Júnior ${ }^{1}$ \\ CECE/UNIOESTE, Foz do Iguaçu, PR \\ Aurelio Ribeiro Leite de Oliveira ${ }^{2}$ \\ IMECC/UNICAMP, Campinas, PR
}

\begin{abstract}
Resumo. Dada a importância e a dificuldade em se obter resultados satisfatórios via métodos diretos para solução de problemas lineares discretos mal-postos oriundos da discretização de problemas inversos lineares. Neste trabalho, nós retomamos o método de pontos interiores do tipo Preditor-Corretor apresentado em [5] que aproxima o problema de regularização de Tikhonov por um problema de programação quadrática através de uma formulação Primal-Dual com barreira logarítmica. Este método Preditor-Corretor nos leva a sistemas de equações normais que são resolvidos pelo método dos gradientes conjugados precondicinado com o precondiconador separador. Neste trabalho, a fim de reduzir o número de iterações de pontos interiores e do método dos gradientes conjugados precondicionado [8], propomos a utilização do precondicionador Fatoração Controlada de Cholesky [1].
\end{abstract}

Palavras-chave. Regularização de Tikhonov, Programação Quadrática, Métodos de Pontos Interiores.

\section{Introdução}

A discretização de um problema inverso comumente fornece um sistema linear de equações

$$
A x=b, A \in \mathbb{R}^{m \times n}, x \in \mathbb{R}^{n}, b \in \mathbb{R}^{m}
$$

Quando os valores singulares da matriz dos coeficientes deste sistema se acumulam próximos à origem e decaem gradualmente a zero, isso torna a matriz severamente mal-condicionada. Tais sistemas são frequentemente chamados de problemas lineares discretos e mal-postos [6].

O método de regularização de Tikhonov é um dos mais antigos e mais populares métodos de regularização. Este método aproxima o sistema linear (1) pelo sistema regularizado

$$
\left(A^{T} A+\alpha^{2} I\right) x=A^{T} b,
$$

onde $\alpha \geq 0$ é o parâmetro de regularização que determina a quantidade de regularização e $I$ é o operador identidade.

Em muitos problemas, a matriz $A$ tem muitos valores singulares pequenos, o que acaba acarretando um mal condicionamento da matriz $A$ e consequentemente de $A^{T} A$, visto que os autovalores de $A^{T} A$ são os valores singulares de $A$ elevados ao quadrado. Além disso, em geral o vetor $b$

\footnotetext{
1 emidio.portilho@gmail.com.

2aurelio@ime.unicamp.br.
} 
está contaminado por erros de medidas (ruídos). Neste trabalho, assumiremos que os erros estão restritos ao lado direito do sistema (1), isto é, dado $b$ podemos escrever

$$
b=\bar{b}+\mathbf{e} \quad \bar{b}=A \bar{x},
$$

onde $\bar{b}$ representa os dados exatos não perturbados, $\bar{x}=A^{\dagger} \bar{b}$ representa a solução exata e o vetor e representa os erros nos dados.

A solução $x$ de um problema mal-posto obtida via métodos diretos está com frequência associada a um valor elevado de $\|x\|_{2}$, veja [7]. Em vista disso o método apresentado por Tikhonov e Arsenin em [7] tem por objetivo obter soluções do sistema de equações lineares $A x=b$ com norma pequena, do que é proposto resolver o problema de minimização:

$$
\min _{x}\|x\|_{2}, \text { sujeito a } \quad\|b-A x\|_{2} \leq \mathbf{T}_{0}
$$

onde $\mathbf{T}_{0}$ é o valor máximo da norma do resíduo que estamos dispostos a aceitar. Sendo assim, estamos diante de um problema de minimização com restrições de desigualdade.

No trabalho [5], o problema de minimização (3) foi aproximado por um problema de programação quadrática através de uma formulação Primal-Dual que deu origem a um método Preditor-Corretor capaz de obter uma solução para o problema de minimização (3). Este método Preditor-Corretor da origem a sistemas de equações normais que são resolvidos via método dos gradientes conjugados precondicinado com o precondiconador separador. Neste trabalho, a fim de reduzir o número de iterações de pontos interiores e do método dos gradientes conjugados precondicionado [8], propomos a utilização do precondicionador Fatoração Controlada de Cholesky [1].

\section{Métodos de Pontos Interiores}

Em [5], para desenvolver o método de pontos interiores (MPI) para o problema de regularização de Tikhonov, nós aproximamos o problema de minimização (3) pelo problema (4), que procura soluções com propriedades similares as requeridas pelo problema (3):

$$
\begin{cases}\min _{x, u, v} & \frac{\tau}{2}\|x\|_{2}^{2}+e^{T}(u+v) \\ \text { sujeito a } & A x+u-v=b . \\ & (u, v) \geq 0 \text { e } x \in \mathbb{R}^{n}\end{cases}
$$

Trata-se de um problema de programação quadrática com restrições lineares, onde $A$ é uma matriz de posto completo $m \times n, b$ e $x$ são vetores colunas de dimensões apropriadas. Além disso, $e$ é um vetor com todas as entradas iguais a 1 e $u$ e $v$ são variáveis não negativas. O valor $\tau>0$ representa um parâmetro de penalização para valores grandes de $\|x\|_{2}$.

Associado ao problema primal (4), temos o problema de programação quadrática dual:

$$
\begin{cases}\max _{x, y, z, w} & -\frac{\tau}{2}\|x\|^{2}+y^{T} b \\ \text { sujeito a } & \tau x-A^{T} y=0 \\ & e-y-z=0 \\ & e+y-w=0 \\ & (z, w) \geq 0 \text { e } y \in \mathbb{R}^{m}\end{cases}
$$

Aplicando os mesmos passos de [9] aos problemas (4) e (5) obtemos um método de pontos interiores do tipo preditor-corretor cujo a estrutura é dada pelo Algoritmo 1. 


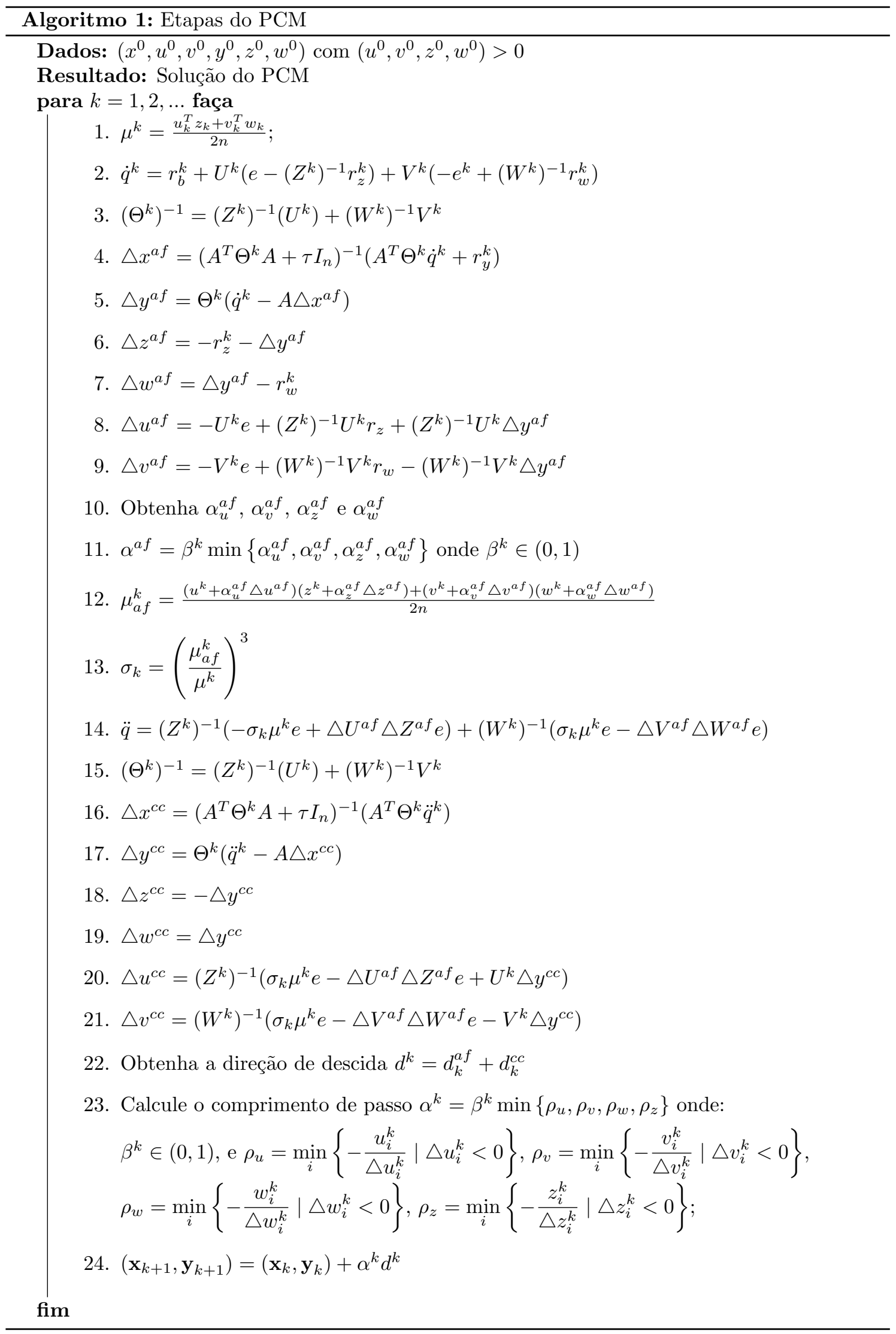


Neste algoritmo valem as seguintes igualdades: $\alpha_{u}^{a f}=\operatorname{argmax}\left\{\alpha \in(0,1]: u^{k}+\alpha \triangle u^{a f}>0\right\}$, $\alpha_{v}^{a f}=\operatorname{argmax}\left\{\alpha \in(0,1]: v^{k}+\alpha \triangle v^{a f}>0\right\}, \alpha_{z}^{a f}=\operatorname{argmax}\left\{\alpha \in(0,1]: z^{k}+\alpha \triangle z^{a f}>0\right\}, \alpha_{w}^{a f}=$ $\operatorname{argmax}\left\{\alpha \in(0,1]: w^{k}+\alpha \Delta w^{a f}>0\right\}, Z^{k}=\operatorname{diag}\left(z_{1}^{k}, z_{2}^{k}, \ldots, z_{m}^{k}\right), U^{k}=\operatorname{diag}\left(u_{1}^{k}, u_{2}^{k}, \ldots, u_{m}^{k}\right)$, $W^{k}=\operatorname{diag}\left(w_{1}^{k}, w_{2}^{k}, \ldots, w_{m}^{k}\right), V^{k}=\operatorname{diag}\left(v_{1}^{k}, v_{2}^{k}, \ldots, v_{m}^{k}\right), r_{b}^{k}=b-A x^{k}-u^{k}+v^{k}, r_{y}^{k}=A^{T} y^{k}-\tau x^{k}$, $r_{z}^{k}=z^{k}+y^{k}-e$ e $r_{w}^{k}=w^{k}-y^{k}-e$.

$\mathrm{Na}$ etapa 4 do Algoritmo 1 nos deparamos com sistemas de equações normais. Para resolver tais sistemas utilizamos o Método dos Gradientes Conjugados Precondicionado (MGCP) [8].

Nós implementamos duas versões do Algoritmo 1, a primeira versão utilizando o precondicionador separador desenvolvido em [3]. Vamos nos referir a esta combinação como MPCLU. A segunda versão foi implementada utilizando a Fatoração Controlada de Cholesky proposta por [1]. A esta combinação iremos nos referir como MPCFCC.

Mais detalhes sobre as formulações MPCLU e MPCFCC podem ser encontras em [4].

\section{Resultados Numéricos}

Todos os resultados numéricos foram desenvolvidos em MATLAB R2013b com sistema operacional 64-bit Windows 10, processador Intel Core I7-8550U, 1.99 Ghz, 16 GB de memória RAM. Os códigos para calcular a discretização dos exemplos deste trabalho provêm dos pacotes disponibilizados em [2]. Consideramos que o lado direito dos sistemas está contaminado por ruídos, ou seja, $b=\bar{b}+\mathbf{e}$, em que e refere-se a um vetor aleatório normalizado escolhido de tal forma que $\|\mathbf{e}\| /\|\bar{b}\|=\epsilon>0$. Iremos nos referir ao quociente $N L=\|\mathbf{e}\| /\|\bar{b}\|$ como nível de ruído. Para os testes numéricos assumimos $\tau=5 \times 10^{-3}$.

A fim de comparar a eficiência do MPCFCC e do MPCLU, iremos comparar os resultados obtidos por ambos os métodos na resolução dos problemas de teste Baart, Shaw e Phillips com níveis de ruído $N L=10^{-3}, N L=10^{-4}$ e $N L=10^{-3}$ respectivamente. Nós testamos ambos os métodos para várias dimensões dos problemas de teste. Apresentamos os resultados nas tabelas a seguir:

Tabela 1: MPCFCC aplicado à matriz de Baart.

\begin{tabular}{rrrrrr}
\hline Dimensão & It & Itgcp & Erd & Eri & $t_{c p u}$ \\
\hline 250 & 8 & 148 & $1,158 \times 10^{-1}$ & $2,403 \times 10^{-4}$ & $2,809 \times 10^{-1}$ \\
\hline 500 & 9 & 129 & $1,201 \times 10^{-1}$ & $1,635 \times 10^{-4}$ & $6,022 \times 10^{-1}$ \\
\hline 1000 & 10 & 131 & $7,760 \times 10^{-2}$ & $5,332 \times 10^{-5}$ & $2,841 \times 10^{+0}$ \\
\hline 2000 & 10 & 131 & $1,058 \times 10^{-1}$ & $4,386 \times 10^{-4}$ & $2,312 \times 10^{+1}$ \\
\hline 5000 & 11 & 147 & $1,101 \times 10^{-1}$ & $2,134 \times 10^{-5}$ & $5,811 \times 10^{+2}$ \\
\hline
\end{tabular}

Tabela 2: MPCLU aplicado à matriz de Baart.

\begin{tabular}{rrrrrr}
\hline Dimensão & It & Itgcp & Erd & Eri & $t_{c p u}$ \\
\hline 250 & 9 & 148 & $1,145 \times 10^{-1}$ & $2,204 \times 10^{-4}$ & $4,127 \times 10^{-1}$ \\
\hline 500 & 11 & 158 & $1,300 \times 10^{-1}$ & $1,657 \times 10^{-4}$ & $5,723 \times 10^{-1}$ \\
\hline 1000 & 10 & 164 & $1,573 \times 10^{-1}$ & $6,239 \times 10^{-5}$ & $1,742 \times 10^{+0}$ \\
\hline 2000 & 12 & 201 & $7,130 \times 10^{-2}$ & $3,557 \times 10^{-5}$ & $8,885 \times 10^{+0}$ \\
\hline 5000 & 10 & 165 & $8,060 \times 10^{-2}$ & $3,120 \times 10^{-5}$ & $5,365 \times 10^{+1}$ \\
\hline
\end{tabular}


Tabela 3: MPCFCC aplicado à matriz de Shaw.

\begin{tabular}{rrrrrr}
\hline Dimensão & It & Itgcp & Erd & Eri & $t_{c p u}$ \\
\hline 250 & 13 & 332 & $3,250 \times 10^{-2}$ & $1,474 \times 10^{-5}$ & $3,200 \times 10^{-1}$ \\
\hline 500 & 13 & 332 & $3,360 \times 10^{-2}$ & $9,887 \times 10^{-6}$ & $7,339 \times 10^{-1}$ \\
\hline 1000 & 14 & 354 & $3,340 \times 10^{-2}$ & $8,180 \times 10^{-6}$ & $3,478 \times 10^{+0}$ \\
\hline 2000 & 14 & 343 & $3,350 \times 10^{-2}$ & $6,793 \times 10^{-6}$ & $2,598 \times 10^{+1}$ \\
\hline 3000 & 15 & 372 & $3,310 \times 10^{-2}$ & $6,273 \times 10^{-7}$ & $8,920 \times 10^{+1}$ \\
\hline
\end{tabular}

Tabela 4: MPCLU aplicado à matriz de Shaw.

\begin{tabular}{rrrrrr}
\hline Dimensão & It & Itgcp & Erd & Eri & $t_{c p u}$ \\
\hline 250 & 12 & 344 & $3,170 \times 10^{-2}$ & $1,283 \times 10^{-5}$ & $4,074 \times 10^{-1}$ \\
\hline 500 & 12 & 337 & $3,450 \times 10^{-2}$ & $1,144 \times 10^{-5}$ & $8,760 \times 10^{-1}$ \\
\hline 1000 & 15 & 396 & $3,230 \times 10^{-2}$ & $5,710 \times 10^{-6}$ & $4,019 \times 10^{+0}$ \\
\hline 2000 & 14 & 356 & $3,310 \times 10^{-2}$ & $7,223 \times 10^{-6}$ & $1,500 \times 10^{+1}$ \\
\hline 3000 & 15 & 382 & $3,360 \times 10^{-2}$ & $6,546 \times 10^{-6}$ & $3,363 \times 10^{+2}$ \\
\hline
\end{tabular}

Tabela 5: MPCFCC aplicado à matriz de Phillips.

\begin{tabular}{rrrrrr}
\hline Dimensão & It & Itgcp & Erd & Eri & $t_{c p u}$ \\
\hline 252 & 9 & 285 & $1,180 \times 10^{-2}$ & $6,052 \times 10^{-4}$ & $2,707 \times 10^{-1}$ \\
\hline 504 & 11 & 377 & $1,440 \times 10^{-2}$ & $5,890 \times 10^{-4}$ & $6,451 \times 10^{-1}$ \\
\hline 1024 & 12 & 417 & $1,480 \times 10^{-2}$ & $3,644 \times 10^{-4}$ & $3,200 \times 10^{+0}$ \\
\hline 2048 & 13 & 502 & $1,210 \times 10^{-2}$ & $4,740 \times 10^{-4}$ & $2,557 \times 10^{+1}$ \\
\hline 4096 & 12 & 458 & $1,120 \times 10^{-2}$ & $4,235 \times 10^{-4}$ & $2,876 \times 10^{+2}$ \\
\hline
\end{tabular}

Tabela 6: MPCLU aplicado à matriz de Phillips.

\begin{tabular}{rrrrrr}
\hline Dimensão & It & Itgcp & Erd & Eri & $t_{c p u}$ \\
\hline 252 & 10 & 385 & $1,000 \times 10^{-2}$ & $3,143 \times 10^{-4}$ & $3,817 \times 10^{-1}$ \\
\hline 504 & 11 & 429 & $1,250 \times 10^{-2}$ & $3,563 \times 10^{-4}$ & $9,546 \times 10^{-1}$ \\
\hline 1024 & 12 & 458 & $9,000 \times 10^{-3}$ & $3,182 \times 10^{-4}$ & $4,547 \times 10^{+0}$ \\
\hline 2048 & 13 & 512 & $1,170 \times 10^{-2}$ & $3,919 \times 10^{-4}$ & $2,302 \times 10^{+1}$ \\
\hline 4096 & 12 & 466 & $1,160 \times 10^{-2}$ & $5,213 \times 10^{-4}$ & $1,038 \times 10^{+2}$ \\
\hline
\end{tabular}


A primeira coluna das tabelas nos informa a dimensão das matrizes, a segunda coluna fornece o número de iterações de métodos de pontos interiores que foram necessárias para resolver o problema, ao passo que a terceira fornece o número de iterações do MGCP necessárias durante toda a execução do método de pontos interiores em questão. Uma vez que a solução exata $\mathbf{x}$ é conhecida, as colunas Erd e Eri representam respectivamente os erros relativos cometidos: Erd $=\left\|\mathbf{x}^{k}-\mathbf{x}\right\|_{2} /\|\mathbf{x}\|_{2}$ e Eri $=\left\|A \mathbf{x}^{k}-b\right\|_{2} /\|b\|_{2}$. Por fim, $t_{\text {cpu }}$ representa o tempo em segundos demandados pelo método para resolução do problema.

Para o problema de Baart as iterações de MPI e iterações de MGCP do método MPCFCC se mostrou competitivo com o método MPCLU, ele também se mostrou competitivo no quesito tempo computacional, exceto para ordem $n=5000$.

Já para o problema de teste Shaw, o número de iterações de ponto interior e de MGCP dos métodos MPCFCC e MPCLU tiveram resultados bem próximos e podemos dizer o mesmo com relação à precisão dos resultados alcançados pelos mesmos. Na maior parte dos casos, o tempo de processamento do MPCFCC obteve resultados melhores do que o método MPCLU.

Por fim, para o problema de Phillips o número iterações de MPI foram os mesmos em quase todos os casos. Mas, o número de iterações de MGCP foram menores em todos os casos. No entanto, os resultados de tempo de processamento $t_{c p u}$ do MPCFCC se tornaram maiores do que os do MPCLU a partir da ordem $n=2048$. Isto se deve à necessidade de atualizar o precondicionador Fatoração Controlada de Cholesky quando o mesmo perde eficiência.

\section{Conclusões}

Neste trabalho, o cálculo das direções de busca do método Preditor-Corretor nos levaram a sistemas de equações normais. Estes sistemas de equações normais foram resolvidos pelo Método dos Gradientes Conjugados Precondicionado. Sendo os precondicionadores utilizados o Precondicionador Separador e a Fatoração Controlada de Cholesky.

De modo geral podemos dizer que o MPCLU e o MPCFCC obtiveram ordem de precisão das soluções bastante similares. Também, o número de iterações de MPI de ambos os métodos se mostraram bem próximos para os exemplos utilizados. No entanto, na maior parte dos casos o método MPCFCC obteve êxito em diminuir o número de iterações de MGCP no decorrer das iterações de MPI.

Concluímos que embora sejam necessários mais testes, os resultados obtidos com a implementação dos métodos no software MATLAB, mostram que o MPCFCC pode ser competitivo com o MPCLU.

\section{Referências}

[1] Campos, F. F. Analysis of conjugate gradient-type methods for solving linear equations, Tese deDoutorado, University of Oxford, 1995.

[2] Hansen, P. C. Regularization tools version 4.0 for matlab 7.3. Numer. Algorithms, volume 46, pages 189-194, 2007. DOI: 10.1007/s11075-007-9136-9.

[3] Oliveira, A. R. L.; Sorensen, D. A new class of preconditioners for large-scale linear systems from interior point methods for linear programming, Linear Algebra and its applications, Elsevier, volume 394, pages 1-24, 2005. DOI: 10.1016/j.laa.2004.08.019.

[4] Portilho Jr, E. S. Métodos de pontos interiores para resolução de problemas de regularização de Tikhonov de grande porte, Tese de Doutorado, Unicamp, 2020. 
[5] Portilho Jr, E. S.; Oliveira, A. R. L. Métodos de pontos interiores para resolução de problemas de regularização de Tikhonov de grande porte, Anais do X Encontro Regional de Matemática Aplicada e Computacional do Rio Grande do Sul - ERMAC-RS, 2020. ISBN: 978-65-5623103-7

[6] Rezghi, R. and Hosseini, S. M. A new variant of l-curve for tikhonov regularization, Journal of Computational and Applied Mathematics, Elsevier, volume 231, pages 914-924, 2009. DOI: 10.1016/j.cam.2009.05.016.

[7] Tikhonov, A. N.; Aarsenin, V. Y. Solution of ill-posed problems. V.H. Winston \& Sons, Washington DC, 1977.

[8] Trefethen, L. N.; Bau, D. Numerical Linear Algebra. Society for Industrial and Applied Mathematics, 1997.

[9] Zhang, Y. A primal-dual interior point approach for computing $l_{1}$ and $l_{\infty}$ solutions of overdetermined linear systems, J. Optim. Theory Appl., volume 77(2), pages 323-341, 1993. DOI:10.1007/BF00940715. 